\title{
A New Blind Adaptive Interference Suppression Scheme for Acquisition and MMSE Demodulation of DS/CDMA Signals
}

Ho-Chi Hwang, Member, IEEE, and Che-Ho Wei, Fellow, IEEE

\begin{abstract}
In this paper, we present an efficient blind algorithm for estimating the code timing of a desired user in an asynchronous direct-sequence code-division multiple-access (DS/CDMA) system over frequency-nonselective-fading channels. The proposed algorithm acquires the code timing explicitly and results in a near-far resistant minimum mean-squared error (MMSE) demodulation without requiring the knowledge of the timing information, amplitudes, and transmitted symbols of all transmissions. The only required knowledge is the information of the signature sequence used by the desired transmission. Several computer simulations are done for additive white Gaussian channels, Rayleigh fading channels, and two-ray Rayleigh fading multipath channels, respectively. Numerical results show that the new algorithm is near-far resistant to the multiple-access interference (MAI) in the DS/CDMA system.
\end{abstract}

Index Terms-Acquisition, code-division multiple access, MMSE demodulation, multiple-access interference.

\section{INTRODUCTION}

$\mathbf{I}$ N AN ASYNCHRONOUS direct-sequence code-division multiple-access (DS/CDMA) communication system, each user transmits the antipodal symbol modulated upon a unique spreading sequence [1]. The conventional matched correlator demodulates the transmitted symbol of the desired user by correlating the received signal with a synchronized replica of the spreading signal of interest. Since the signal vectors of all users are not completely orthogonal, the matched correlator is vulnerable to the so-called near-far problem, and thus the system capacity is limited by the multiple-access interference (MAI). The minimum mean-squared error (MMSE) linear detector [2]-[4] has been proposed to suppress the multiuser interference and overcome the near-far problem by utilizing the cyclostationarity of the highly structured MAI. This MMSE linear detector can be implemented using the training-sequence-based adaptation algorithms [3] or the blind adaptation algorithms [5]-[8].

Demodulation of a DS/CDMA signal must be preceded by timing acquisition, which achieves the initial coarse alignment of the local symbol clock within one chip of the incoming symbol clock [9]. In this paper, we develop an efficient al-

Manuscript received December 9, 1997; revised March 9, 1999. This work was supported by the National Science Council of the Republic of China under Grant NSC87-2221-E-009-001.

H.-C. Hwang is with the Computer \& Communications Research Laboratories, Industrial Technology Research Institute, Taiwan.

C.-H. Wei is with the Department of Electronics Engineering, National Chiao Tung University, Hsin-Chu, Taiwan, R.O.C. (e-mail: chwei@cc.nctu.edu.tw).

Publisher Item Identifier S 0018-9545(00)02573-1. gorithm for estimating the code timing of a specific desired user without requiring a training sequence. The proposed algorithm [called projection degree measurement (PDM) algorithm] quantizes the timing delay uncertainty of interest into a finite set of hypotheses, measures the projection degree of a nominal desired signal vector in the signal subspace of the correlation matrix for each hypothesis, and picks the best hypotheses to estimate the time delay. After the code timing is estimated explicitly, the MMSE demodulator is obtained via the Wiener-Hopf equation. The new algorithm is shown to be robust to the near-far problem, and its performance compares favorably to other recently proposed near-far resistant code timing estimations schemes [10]-[15].

\section{THEORETICAL BACKGROUND}

In a DS/CDMA communication environment with $K$ active users, the received signal from the $k$ th user is given by

$$
U_{k}(t)=\sum_{j=-\infty}^{\infty} A_{k} b_{k}[j] s_{k}\left(t-j T_{b}-\tau_{k}\right) \cos \left(\omega_{c} t+\theta_{k}\right)
$$

where $\omega_{c}$ and $T_{b}$ are the carrier frequency and the symbol interval common to all users, respectively. The $j$ th antipodal symbol $b_{k}[j] \in\{+1,-1\}$ of the $k$ th user is transmitted with amplitude $A_{k}$. The time delay $\tau_{k}$ and the carrier phase $\theta_{k}$ relative to the receiver are randomly distributed over $\left[0, T_{b}\right]$ and $[0,2 \pi]$, respectively. To share the same frequency spectrum simultaneously, each user is assigned with a unique spreading waveform given by

$$
s_{k}(t)=\sum_{n=0}^{N-1} a_{k}[n] \Pi\left(t-n T_{c}\right)
$$

where $a_{k}[n] \in\{+1,-1\}$ is the $n$th element of the signature sequence for the $k$ th user and the chip waveform $\Pi(t)$ is a rectangular waveform of unit amplitude and duration $T_{c}$. Without loss of generality, $T_{c}$ is set equal to $T_{b} / N$ and $N$ is the processing gain.

Assuming a frequency-nonselective-fading channel, the received signal is of the form

$$
r(t)=\sum_{k=1}^{K} f_{k}(t) U_{k}(t)+x(t) .
$$

In the above expression, $f_{k}(t)$ is a complex fading factor expressing the amplitude and phase of the fading process for the 
$k$ th user, while $x(t)$ is the complex additive white Gaussian noise (AWGN) with a spectral density of $N_{o}$. In this paper, it is assumed that the reverse channel (i.e., mobile to base station) of a CDMA network is considered, in which the fading processes for all active users are independent and uncorrelated. Furthermore, it is assumed that the fading process for each user varies at a slow rate so that the amplitude and phase are taken to be constant over a bit interval [4]. Throughout this paper, the first user is taken to be the desired user.

Assuming that the receiver has knowledge of the carrier phase of the desired signal, $\theta_{1}$ can be set to zero without loss of generality. The receiver front-end consists of a mixer to convert the received signal to complex baseband followed by a filter matched to the chip pulse shape (i.e., an integrate-and-dump filter with integration time $T_{c}$ since a rectangular chip waveform is used here). The output of this matched filter is then sampled at the chip rate. For capturing the complete information of one desired data symbol, the minimum length of the observation interval is chosen to be $2 T_{b}$. Therefore, the samples within the $j$ th observation interval $\left[j T_{b},(j+2) T_{b}\right)$ can be expressed as a vector

$$
\begin{aligned}
\mathbf{r}(j)= & \sum_{k=1}^{K} b_{k}[j-1] f_{k}(j-1) \mathbf{v}_{k}^{-1}+b_{k}[j] f_{k}(j) \mathbf{v}_{k}^{0} \\
& +b_{k}[j+1] f_{k}(j+1) \mathbf{v}_{k}^{+1}+\mathbf{x}(j)
\end{aligned}
$$

where

$$
\begin{aligned}
\mathbf{a}_{k} & =\left[a_{k}[0], \cdots, a_{k}[N-1], \mathbf{o}_{N}^{T}\right]^{T} \\
\mathbf{v}_{k}^{-1} & =A_{k}\left\{\left(1-\delta_{k}\right) T_{L}^{N-n_{k}} \mathbf{a}_{k}+\delta_{k} T_{L}^{N-n_{k}-1} \mathbf{a}_{k}\right\} \\
\mathbf{v}_{k}^{0} & =A_{k}\left\{\left(1-\delta_{k}\right) T_{R}^{n_{k}} \mathbf{a}_{k}+\delta_{k} T_{R}^{n_{k}+1} \mathbf{a}_{k}\right\} \\
\mathbf{v}_{k}^{+1} & =A_{k}\left\{\left(1-\delta_{k}\right) T_{R}^{n_{k}+N} \mathbf{a}_{k}+\delta_{k} T_{L}^{n_{k}+N+1} \mathbf{a}_{k}\right\}
\end{aligned}
$$

In the above expressions, since $\tau_{k} \in\left[0, T_{b}\right), 0 \leq n_{k} \leq N-1$, and $\delta_{k} \in[0,1)$ are the integral and decimal parts of the delay $\tau_{k}$, respectively, that is, the delay for the $k$ th user relative to the receiver can be written as $\tau_{k}=\left(n_{k}+\delta_{k}\right) T_{c}$. In addition, $f_{k}(j)$ is the fading process for the $k$ th user during the $j$ th bit interval, $\mathbf{0}_{N}$ is a vector of $N$ zeros, and $T_{L}^{n}$ and $T_{R}^{n}$ are the acyclic $n$-shift operators, which acyclically shift the elements of a vector to left and right, respectively. For the sake of simplicity, the $\cos \left(\theta_{k}\right)$ terms are absorbed into $A_{k}$.

In order to suppress the MAI, the MMSE linear detector [2]-[4] demodulates the transmitted symbol of the desired transmission as $\hat{b}_{1}(j)=\operatorname{sgn}\left(\operatorname{Re}\left\{\mathbf{w}^{H} \mathbf{r}(j)\right\}\right)$, where $H$ denotes the complex conjugate and the FIR filter $\mathbf{w}$ minimizes the mean-squared error between the desired symbol and the decision statistic

$$
J=E\left\{\left|b_{1}[j]-\mathbf{w}^{H} \mathbf{r}(j)\right|^{2}\right\} .
$$

The unique optimum tap-weight vector is given by $\mathbf{w}_{o}=$ $\mathbf{R}^{-1} \mathbf{P}$, where $\mathbf{R}=E\left\{\mathbf{r}(j) \mathbf{r}^{H}(j)\right\}$ and $\mathbf{P}=E\left\{b_{1}[j] \mathbf{r}(j)\right\}$ are the correlation matrix of the received signal vector and the correlation between the desired symbol and the received signal vector, respectively.

\section{Projection Degree Measurement Algorithm}

It is convenient to describe the asynchronous DS/CDMA channel by an equivalent synchronous model, in which the received signal vector can be expressed by

$$
\mathbf{r}(j)=b_{1}[j] f_{1}(j) \mathbf{u}_{1}+\sum_{l=2}^{L} b_{l}[j] f_{l}(j) \mathbf{u}_{j}+\mathbf{x}(j) .
$$

In the above expression, $b_{1}[j]$ is the desired symbol modulated with the desired signal vector $\mathbf{u}_{1} \in R^{2 N \times 1}, b_{l}[j]$, for $2 \leq l \leq$ $L$, are interfering symbols due to intersymbol interference and MAI, and $\mathbf{u}_{l} \in R^{2 N \times 1}$ are the interference vectors modulating these symbols. $f_{l}(j)$ is the fading process for the $l$ th user at the $j$ th observation interval. Apparently, the number of virtual users is given by $L=3 K$.

Assuming that the data symbols $b_{l}[j] \in\{+1,-1\}$ are independent and uncorrelated. The correlation matrix $\mathbf{R}$ of the received signal vectors can be expressed as

$$
\mathbf{R}=\mathbf{U}^{T}+\sigma^{2} \mathbf{I}
$$

where $\mathbf{U}=\left[\rho_{1} \mathbf{u}_{1}, \cdots, \rho_{L} \mathbf{u}_{L}\right]$ is the signal matrix with $\rho_{7}^{2}=$ $E\left\{\left|f_{l}(j)\right|^{2}\right\}$ and $\sigma^{2}$ is the noise variance. The correlation matrix can be further expressed in terms of its eigendecomposition as

$$
\mathbf{R}=\mathbf{Q D Q}^{T}
$$

where the columns $\mathbf{q}_{n}, n=1, \cdots, 2 N$, of $\mathbf{Q} \in R^{2 N \times 2 N}$ are the eigenvectors of $\mathbf{R}$ and $\mathbf{D} \in R^{2 N \times 2 N}$ is a diagonal matrix with eigenvalues $\lambda_{n}$. If the number of virtual users is less than the dimension of the received signal vector (i.e., $L<2 N$ ), we have $\lambda_{1} \geq \lambda_{2} \geq \cdots \geq \lambda_{2 N}$ and

$$
\lambda_{n}= \begin{cases}\gamma_{n}+\sigma^{2}, & \text { if } n \leq L \\ \sigma^{2}, & \text { otherwise }\end{cases}
$$

where $\gamma_{n}, n=1, \cdots, L$ and $\gamma_{1} \geq \gamma_{2} \geq \cdots \geq \gamma_{L}$ denote the eigenvalues of $\mathbf{U U}^{T}$.

The matrix $\mathbf{Q}$ can be partitioned as $\mathbf{Q}=\left[\mathbf{Q}_{s} \mathbf{Q}_{n}\right]$, in which the columns of $\mathbf{Q}_{s}=\left[\mathbf{q}_{1}, \cdots, \mathbf{q}_{L}\right]$ are associated with the $L$ largest eigenvalues and form a basis for the signal subspace, and the columns of $\mathbf{Q}_{n}=\left[\mathbf{q}_{L+1}, \cdots, \mathbf{q}_{2 N}\right]$ are associated with the remaining eigenvalues and span the noise subspace. Due to the orthogonal property between the eigenvectors, the signal subspace is the orthogonal complement of the noise subspace. All the eigenvectors associated with the $(2 N-L)$ smallest eigenvalues of $\mathbf{R}$ must satisfy the following:

$$
\left(\mathbf{R}-\sigma^{2} \mathbf{I}\right) \mathbf{q}_{p}=\mathbf{0}, \quad p=L+1, \cdots, 2 N .
$$

If the signal matrix $\mathbf{U}$ is assumed to be of full column rank $L$, it follows from the above equation that

$$
\begin{array}{ll}
\mathbf{u}_{l}^{T} \mathbf{q}_{p}=0, \quad l=1, \cdots, L \\
& p=L+1, \cdots, 2 N .
\end{array}
$$

Consequently, the signal vectors, $\mathbf{u}_{l}, l=1, \cdots, L$, are orthogonal to the noise subspace. According to the properties of eigendecomposition, we obtain the following lemma, which is proved in Appendix A. 
1) Lemma: Define a Hermitian matrix $\mathbf{R} \in R^{2 N \times 2 N}$ as $\mathbf{R}=\mathbf{U} \mathbf{U}^{T}+\sigma^{2} \mathbf{I}$, where $\mathbf{U}=\left[\mathbf{u}_{1}, \cdots, \mathbf{u}_{L}\right]$ is the signal matrix, $\mathbf{u}_{l} \in R^{2 N \times 1}$ and $l=1, \cdots, L$ are the signal vectors, and $\sigma^{2}$ is the noise variance. Let $\mathbf{c} \in R^{2 N \times 1}$ be a vector, which does not lie completely in the signal subspace of $\mathbf{R}$. If $L<2 N$ and $\sigma^{2}$ is small enough, the following relation holds:

$$
\frac{\mathbf{c}^{T} \mathbf{R}^{-1} \mathbf{c}}{|\mathbf{c}|^{2}} \geq \frac{\mathbf{u}_{l}^{T} \mathbf{R}^{-1} \mathbf{u}_{l}}{\left|\mathbf{u}_{l}\right|^{2}}
$$

The lemma states that a large value of $\mathbf{c}^{T} \mathbf{R}^{-1} \mathbf{c} /|\mathbf{c}|^{2}$ is produced if $\mathbf{c}$ has a significant projection component in the noise subspace of $\mathbf{R}$. By utilizing this lemma, we quantize coarsely the delay of interest into a number of hypotheses and obtain the corresponding nominal desired signal vectors

$$
\begin{aligned}
& H_{2 i-1}: \quad \tau_{1,2 i-1}=(i-1.0) T_{c} \quad \mathbf{u}_{1,2 i-1}=\frac{T_{R}^{i-1} \mathbf{a}_{1}}{\left\|T_{R}^{i-1} \mathbf{a}_{1}\right\|} \\
& H_{2 i}: \quad \tau_{1,2 i}=(i-0.5) T_{c} \quad \mathbf{u}_{1,2 i}=\frac{T_{R}^{i-1} \mathbf{a}_{1}+T_{R}^{i} \mathbf{a}_{1}}{\left\|T_{R}^{i-1} \mathbf{a}_{1}+T_{R}^{i} \mathbf{a}_{1}\right\|}, \\
& \quad i=1, \cdots, N .
\end{aligned}
$$

Then, the projection degree of the nominal desired signal vector under each hypothesis is measured and the best delay hypotheses are chosen for estimating the delay.

The PDM algorithm is summarized by the following steps.

Step 1) Compute the estimated correlation matrix ${ }^{1}$

$$
\hat{\mathbf{R}}=\frac{1}{M} \sum_{j=1}^{M} \mathbf{r}(j) \mathbf{r}^{H}(j) .
$$

Step 2) Evaluate the projection degree measurement for each nominal desired signal vector $\mathbf{u}_{1, n}$

$$
\xi_{n}=\mathbf{u}_{1, n}^{T} \hat{\mathbf{R}}^{-1} \mathbf{u}_{1, n}, \quad n=1, \cdots, 2 N .
$$

Step 3) Find the best hypotheses

$$
n_{\min }=\min \left\{\xi_{n}\right\}
$$

and

$$
n_{\text {next }}= \begin{cases}2, & n_{\min }=1 \\ 2 N-1, & n_{\min }=2 N \\ \min \left\{\xi_{n_{\text {min }}-1}, \xi_{n_{\min }+1}\right\}, & \text { otherwise }\end{cases}
$$

Step 4) Determine $\hat{\tau}_{1}$ by solving a quadratic function, as described in Appendix B.

\section{NUMERICAL RESULTS}

We consider a symbol- and chip-asynchronous DS/CDMA communication system in different channels. Each user is modulated upon a unique Gold code with length $N=15$. The signal-to-noise ratio (SNR) is defined as SNR $=A_{1}^{2} N / \sigma^{2}$. The numerator $A_{1}^{2} N$ is merely the bit energy $E_{b}$, and $\sigma^{2}=N_{o} / 2$,

\footnotetext{
${ }^{1} \mathrm{~A}$ recursive least squares implementation might also be used in order to track time variations.
}

so that $\mathrm{SNR}(\mathrm{dB})=E_{b} / N_{o}(\mathrm{~dB})+3$. Throughout all experiments, each interfering user is assumed to have the same power level for the sake of simplicity. The near-far ratio (NFR) is used to present the interference level of each interfering user relative to the desired user. The delays and the transmitted symbols for all active users are randomly generated and unknown to the receiver in every simulation run.

\section{A. Acquisition Performance}

The acquisition scheme estimates the code timing close enough such that the code tracking loop can work on driving the timing error to zero. If the estimation timing error is in excess of $\pm 0.5 T_{c}$, an acquisition error happens. We use 1000 simulation runs to estimate the average acquisition error probability and root mean-squared error (RMSE), in which the error is the difference between the estimation and exact timing when the acquisition is correct. The RMSE is normalized with respect to $T_{c}$ in the following. Three other timing estimation techniques: MUSIC estimator [11], MOE estimator [14], and LSML estimator [15] are also investigated for comparison. The different aspects among the PDM algorithm and three other timing estimation techniques are briefly described below. In the LSML estimator that requires a training sequence for the desired user, it is assumed that the transmitter and receiver have aligned their clocks roughly within a bit interval with the aid of a side "signaling channel," where a call is initially set up [15]. The major drawbacks of the blind MUSIC estimator are that a singular value decomposition is necessary and the number of users must limit to below $N / 2$ when one sample is taken per chip interval and one antenna is used. The blind MOE estimator requires the fictitious noise variance $\nu=\alpha \cdot \operatorname{trace}(\hat{\mathbf{R}})$ [14] to obtain the MOE solutions. Although the method for automatically choosing this factor is available, it is not completely satisfactory due to its high complexity. Furthermore, two cost functions, MOE and normalized MOE, are required in the acquisition process. Note that the MOE estimator is implemented without constraints when $\alpha=0$. The observation interval is $T_{b}$ for the MUSIC and LSML estimators, while $2 T_{b}$ for the MOE and PDM estimators.

Figs. 1 and 2 show the acquisition error probability and normalized RMSE as a function of the number of observation vectors $M$ for AWGN channels. The simulation parameters are $K=6, \mathrm{SNR}=20 \mathrm{~dB}$, and $\mathrm{NFR}=20 \mathrm{~dB}$. The value $\alpha$, controlling the fictitious noise variance, is set as $\alpha=0.0001$. Since the sample correlation matrix must be full rank in order to form its inverse matrix, the number of observation vectors cannot be less than $N$ for the LSML estimator, while $2 N$ for the MOE estimator without constraints and the PDM estimator. The plots show that the LSML estimator can acquire the code timing in 1000 simulation runs without any acquisition error as long as the number of the observation vectors increase beyond 50. Furthermore, its normalized RMSE is the least one among them. Similar to the LSML estimator, the MUSIC, MOE and PDM estimators are robust to the near-far problem and offer similar performance in terms of probability of acquisition error and RMSE. However, as the number of observation vectors increase, the performance of these blind estimators converges to a floor. Taking a close look at the plots, in terms of the acquisition 


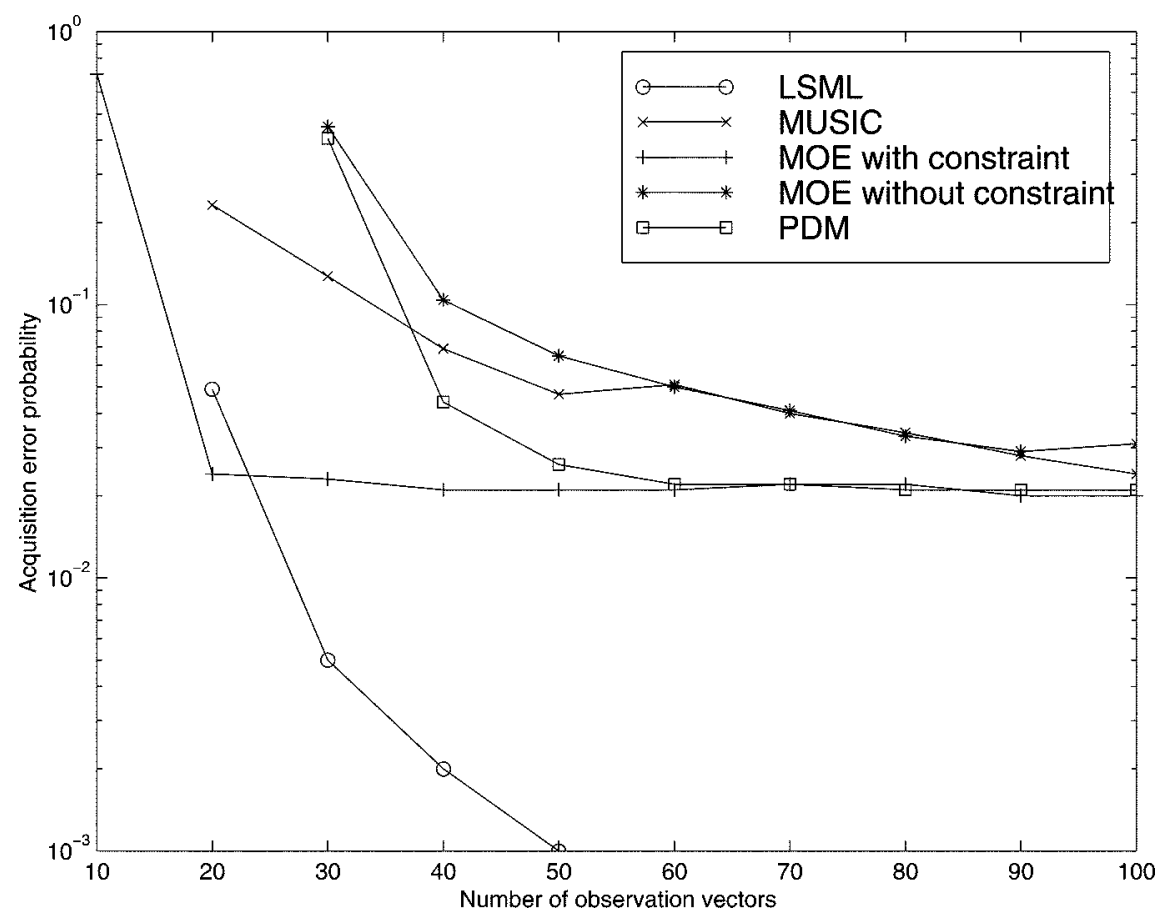

Fig. 1. Acquisition error probability versus the number of observation vectors.

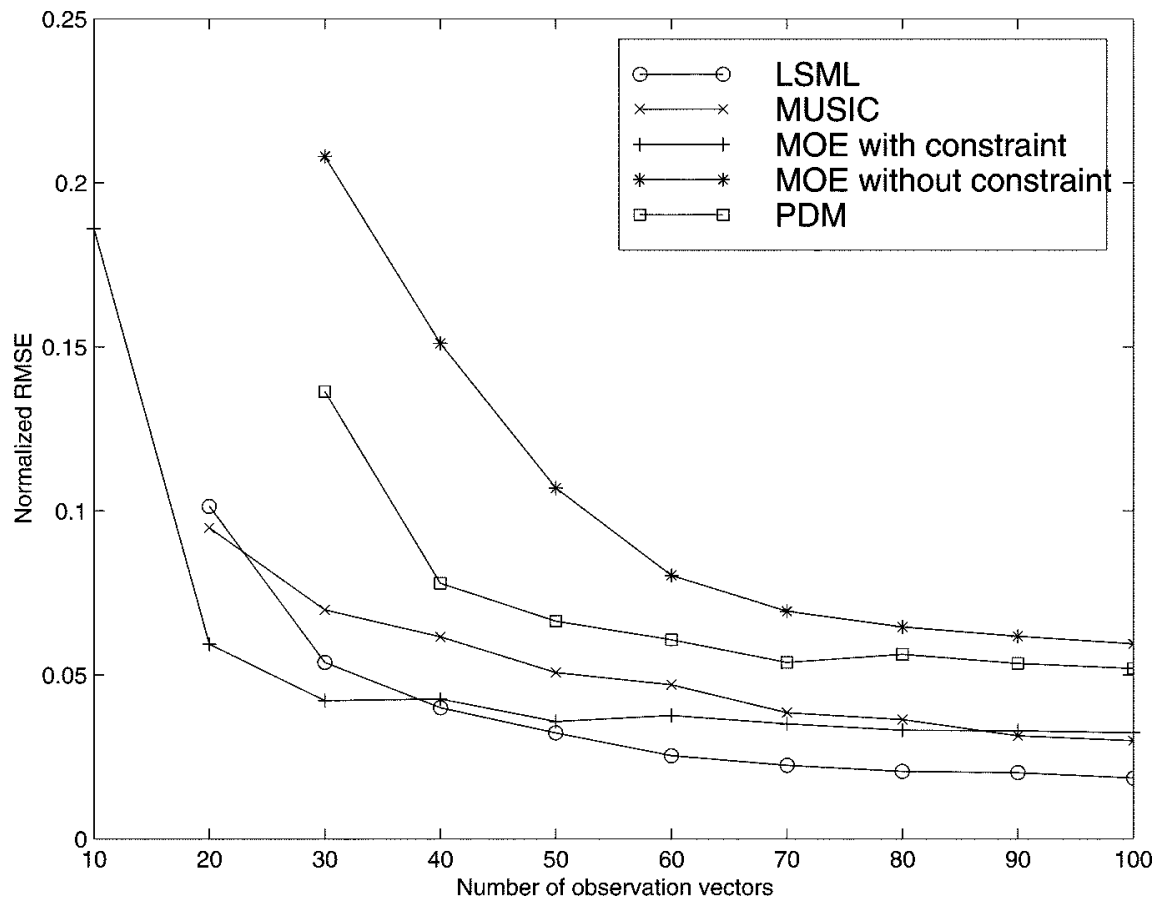

Fig. 2. Normalized RMSE versus the number of observation vectors.

error probability, the MUSIC estimator and the MOE estimator without constraints are slightly worse.

In the next experiment, the performance of the estimators, as shown in Figs. 3 and 4, is investigated versus the number of active users for AWGN channels. The number of observation vectors is $M=100$ and $K$ is varied from 5 to 15 . All other parameters are as before. The MUSIC estimator cannot function when $K>8$. At the same acquisition error probability level (i.e., acquisition error probability of 0.1 ), the PDM estimator and the MOE estimator without constraints can provide a load of 11 users, while the LSML estimator and the MOE estimator with constraints for 9 and 10 users, respectively. Again, the normalized RMSE values of the PDM estimator and the MOE estimator without constraints are smaller in the range of 9-13 users. Although the lemma is imposed by the constraint $3 K<2 N$, the PDM estimator still functions when $K>10$ in this case.

In the third experiment, the performance of the estimators as a function of $E_{b} / N_{o}$ in AWGN channels is compared in Figs. 5 and 6. The number of observation vectors is $M=100$ and five interfering users with NFR $=20 \mathrm{~dB}$ are considered. The 


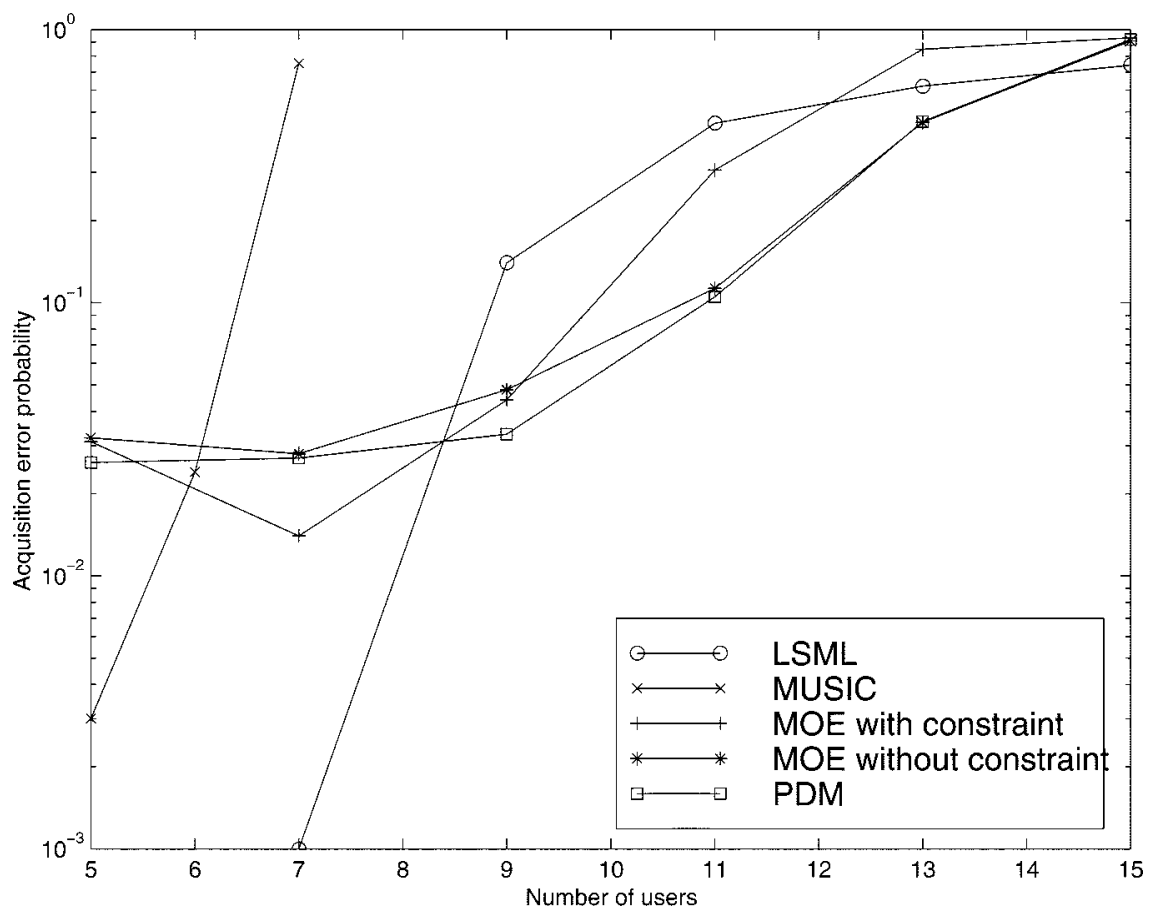

Fig. 3. Acquisition error probability versus the number of users.

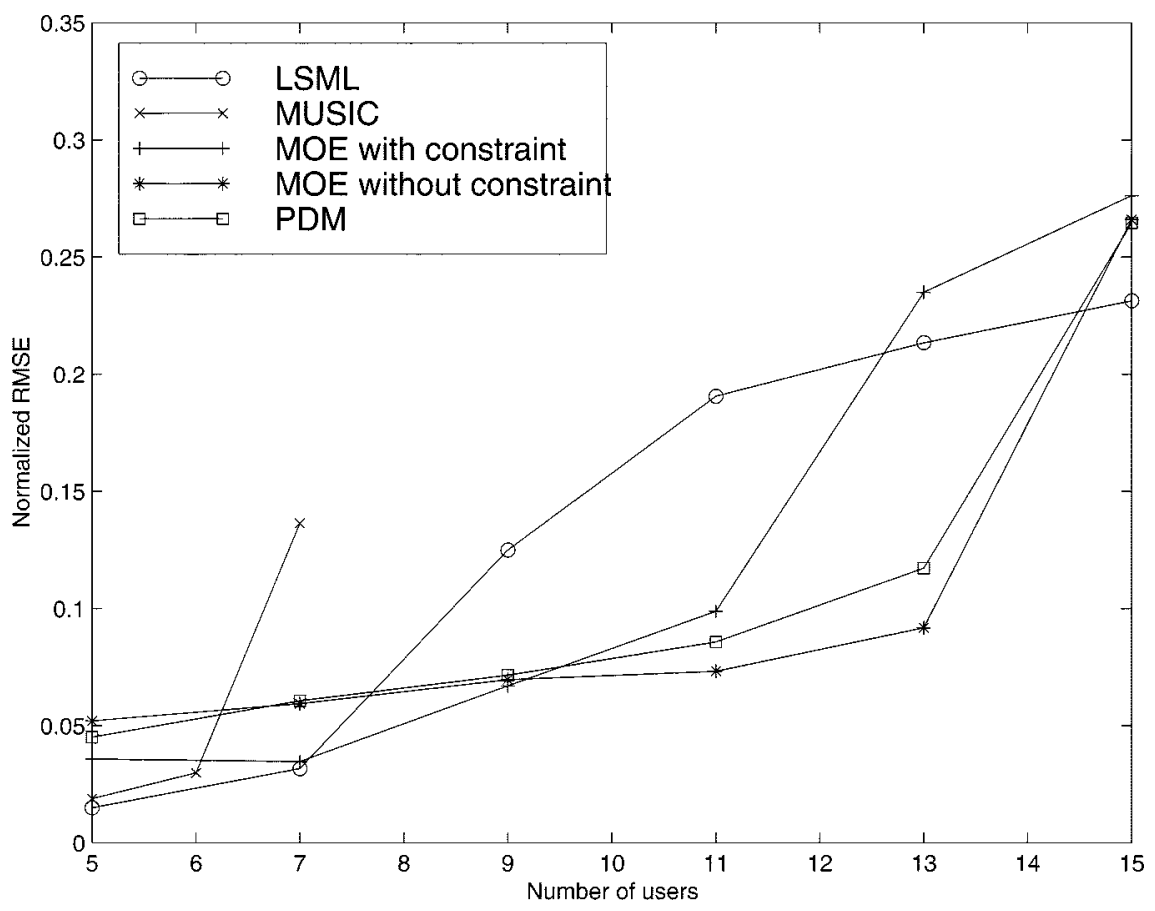

Fig. 4. Normalized RMSE versus the number of users.

LSML estimator performs better than the other estimators because the LSML estimator is train-sequence aided and more robust to AWGN. The performance of the PDM estimator is comparable to the MOE estimator, while the MUSIC estimator is susceptible to noise in terms of acquisition error probability.

In this case, the performance of the estimators is investigated for Rayleigh fading on all received signal with a maximum Doppler frequency (same for all mobiles). In practice the mobile users will move at different velocity and experience different fade rates. This worst case situation is assumed for emphasizing the tracking capability of the estimators. The numbers of observation vectors and active users are $M=100$ and $K=6$, respectively. NFR and SNR are taken to be $20 \mathrm{~dB}$ and $\alpha$ is 0.0001 . When the maximum Doppler frequency is below $50 \mathrm{~Hz}$, the LSML estimator, as shown in Figs. 7 and 8, outperforms the other estimators in terms of acquisition error probability. However, the acquisition performance of the LSML estimator is more susceptible to larger maximum Doppler frequency. On the other hand, the PDM estimator together with the MUSIC and MOE estimators are robust to the variations of fade rates. 


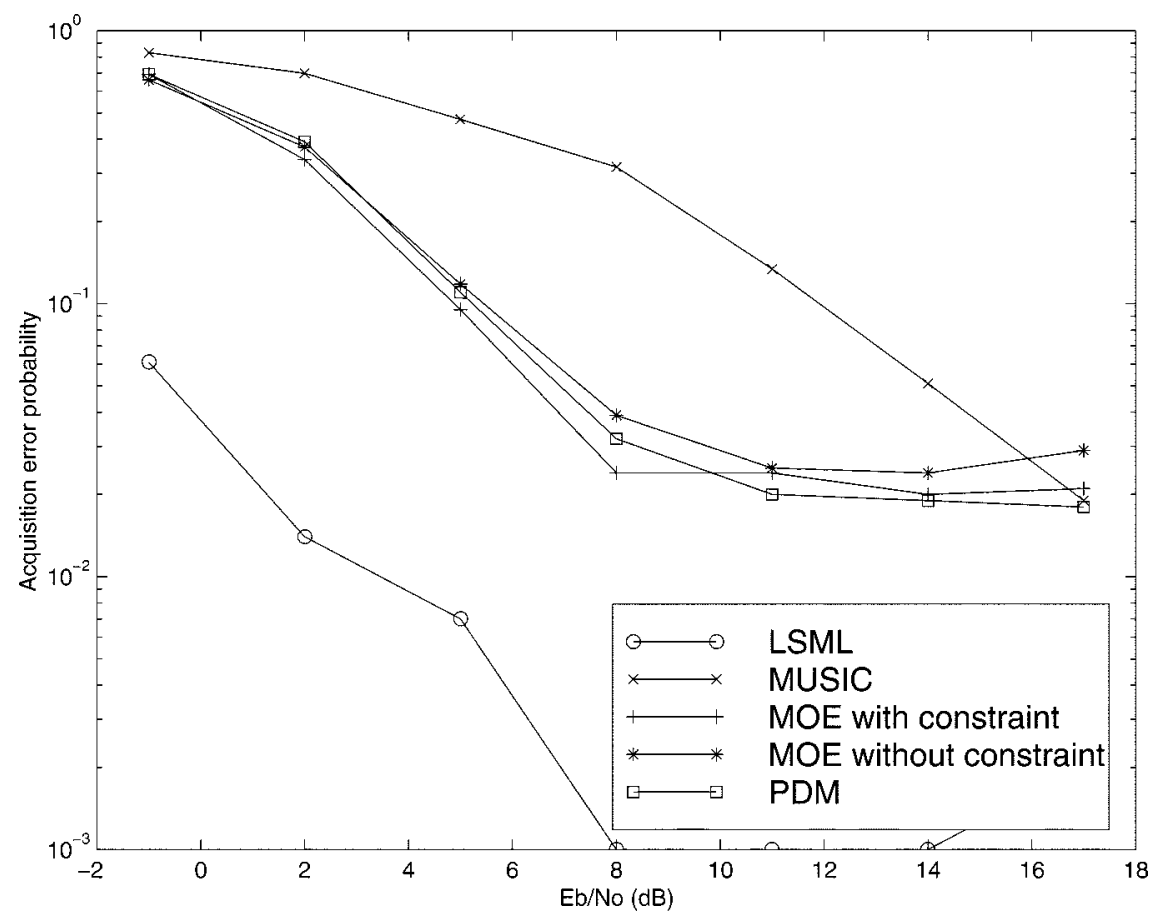

Fig. 5. Acquisition error probability versus SNR.

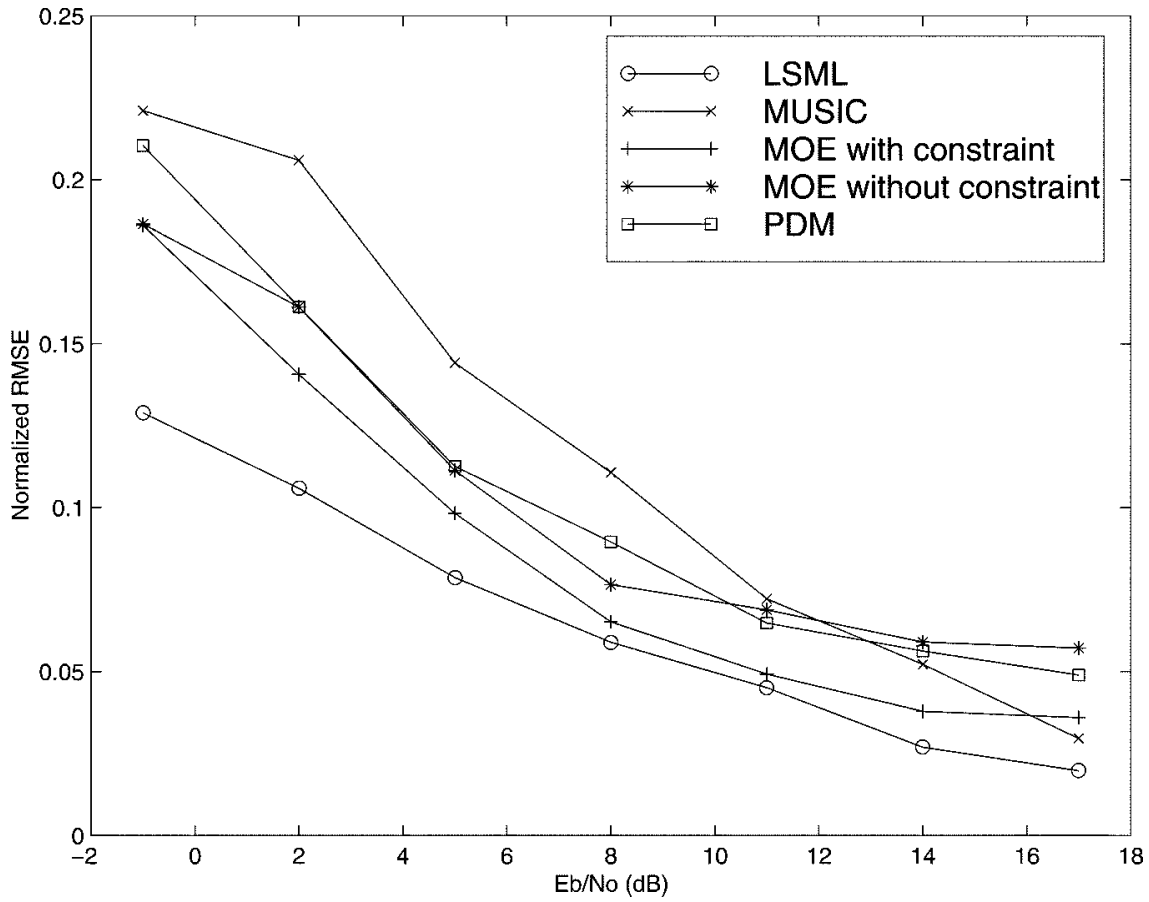

Fig. 6. Normalized RMSE versus SNR.

The multipath effect is considered in this case. All active users are assumed to move at the same velocity $100 \mathrm{~km} / \mathrm{h}$, and the transmitted signal of each user propagates through a unique two-ray Rayleigh fading channel, in which the channel responses of the main and secondary paths are independent and uncorrelated Rayleigh fading processes. The power ratio between the main and secondary paths is denoted as $\Gamma(\mathrm{dB})$. The simulation parameters are $M=100, K=6$, $\mathrm{NFR}=20 \mathrm{~dB}$, and SNR $=20 \mathrm{~dB}$. The value $\alpha$ is as before. The results are obtained from 1000 independent simulation runs and shown in Figs. 9 and 10. An acquisition error happens if the difference between the timing estimate and the timing delay of the main path is in excess of $\pm 0.5 T_{c}$. The MUSIC estimator fails to acquire the code timing in the multipath channel; even the multipath level is low (i.e., $10 \mathrm{~dB}$ ). For the other three timing estimators, their acquisition performance is not acceptable when the multipath interference is strong (i.e., $\Gamma=0 \mathrm{~dB}$ ). As $\Gamma>4 \mathrm{~dB}$, the PDM estimator and the MOE estimator without constraints can provide more than $70 \%$ correct acquisition probability. However, the LSML estimator 


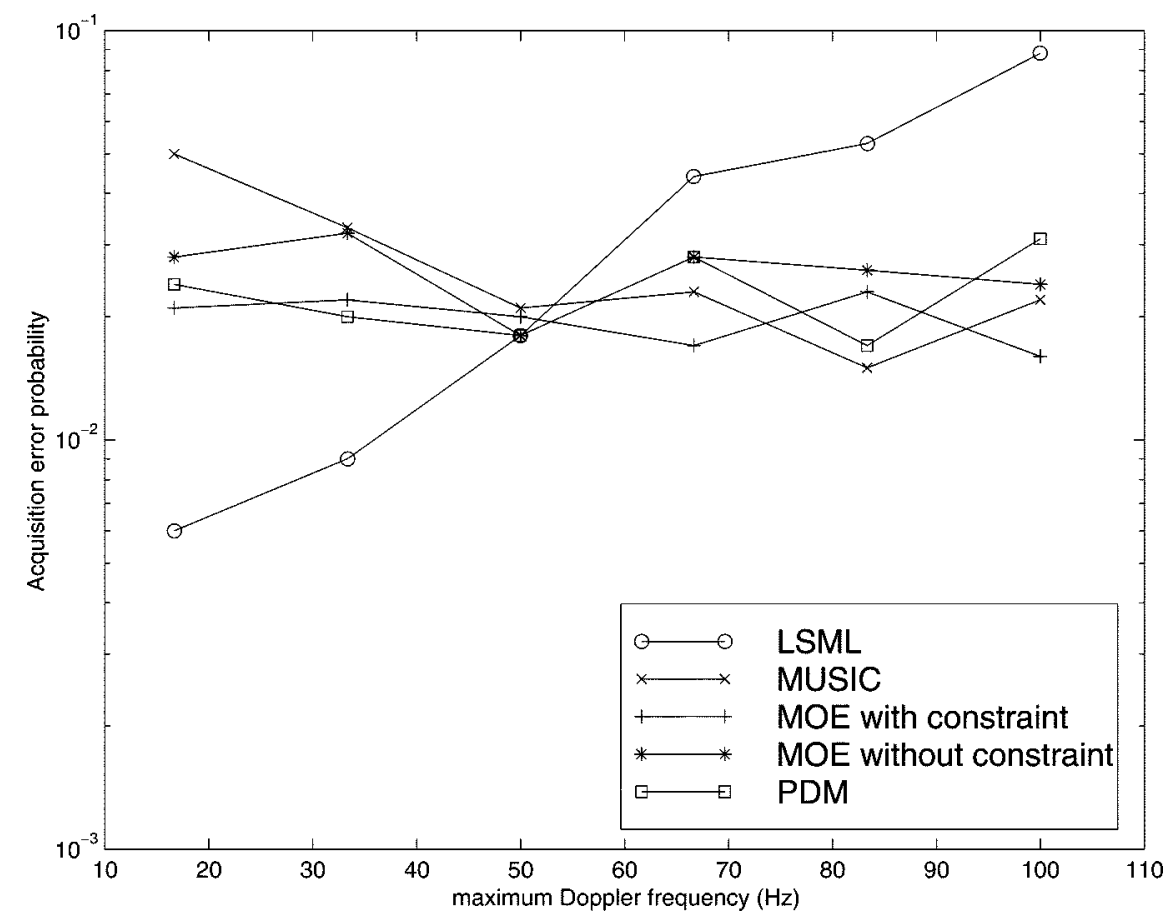

Fig. 7. Acquisition error probability versus maximum Doppler frequency.

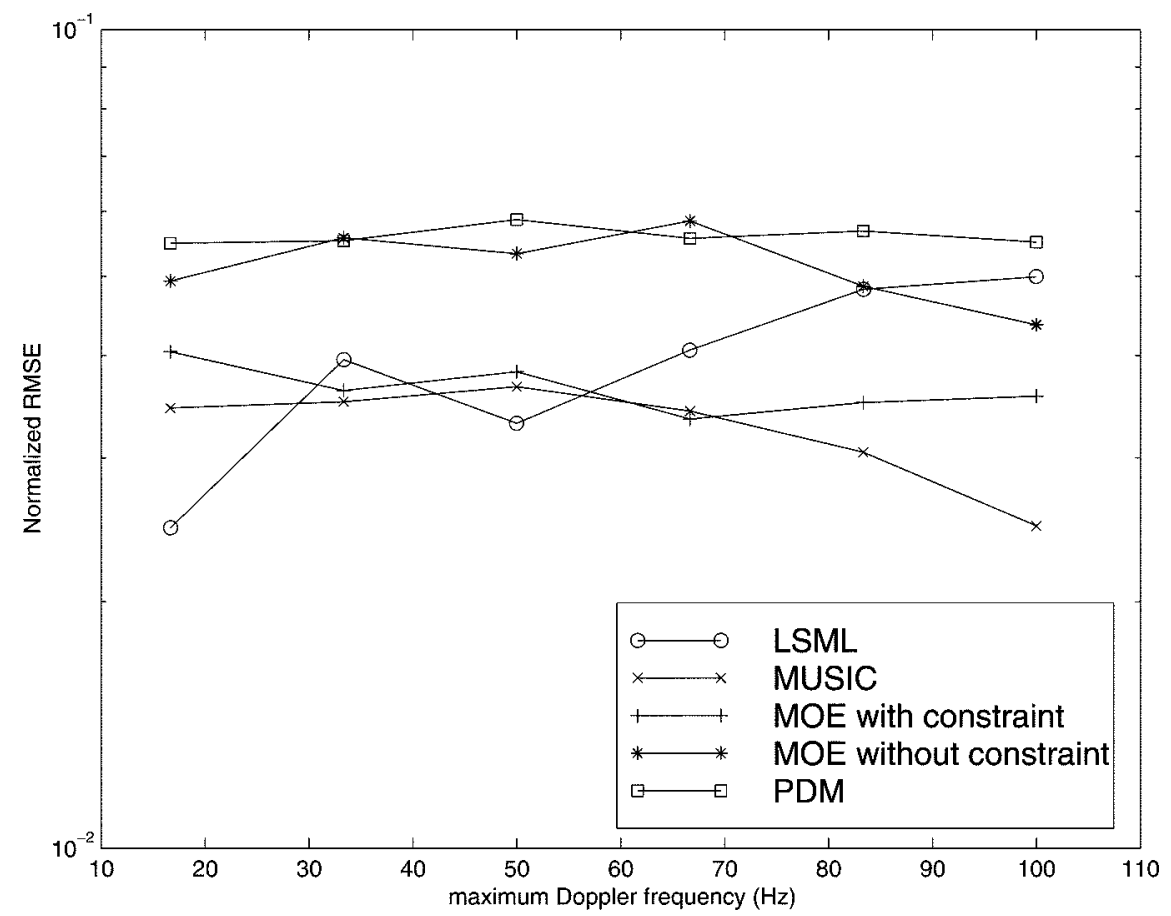

Fig. 8. Normalized RMSE versus maximum Doppler frequency.

and the MOE estimator with constraints have less confidence in exact acquisition.

\section{B. Error Probability Performance}

The bit error probability of the resultant demodulator derived by the PDM algorithm is now evaluated in comparison to that of the MOE algorithm for AWGN channels. ${ }^{2}$ In each simulation, the desired signal vector is determined for forming the demodulator via the Wiener-Hopf solution after the code timing

2In [4], a modified MMSE detector was presented to avoid loss of phase lock during deep fades by incorporating a channel phase estimator to remove the phase variations from the received signal. 


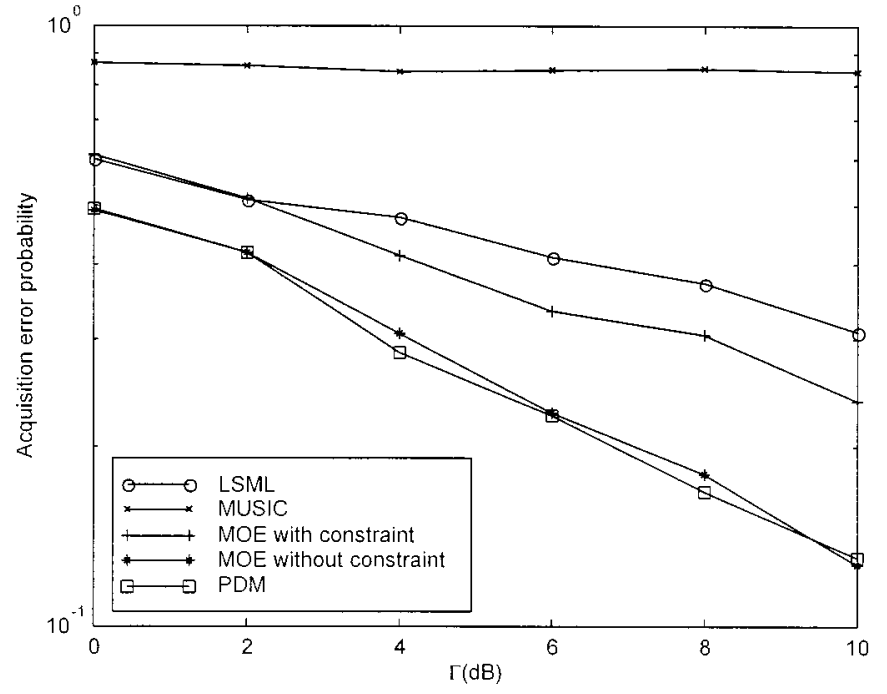

Fig. 9. Acquisition error probability versus $\Gamma$.

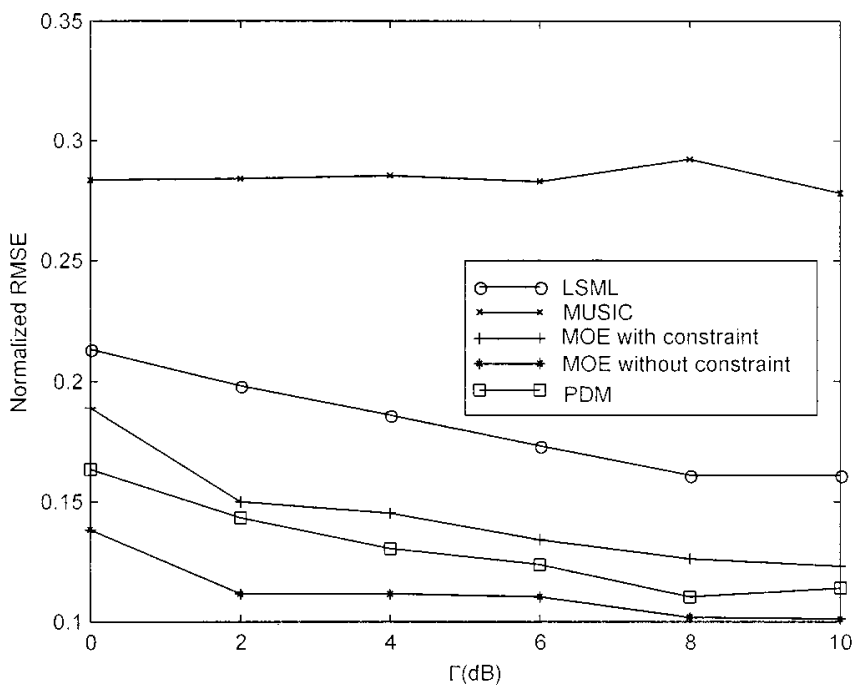

Fig. 10. Normalized RMSE versus $\Gamma$.

is estimated. The error probability is then evaluated analytically using the equation

$$
P_{e}(\mathbf{w})=E\left\{Q\left(\frac{\mathbf{w}^{T} \mathbf{u}_{1}+\sum_{l=2}^{L} b_{l}[j] \mathbf{w}^{T} \mathbf{u}_{l}}{\sigma\|\mathbf{w}\|}\right)\right\}
$$

where the expectation is taken over the interference bits $b_{l}(j), l=2, \cdots, L$, and $Q($.$) is the complementary distribu-$ tion of a standard Gaussian random variable.

The simulation parameters are $N=15, K=6, \mathrm{SNR}=20$ $\mathrm{dB}$, and $M=300$. For a given NFR, 100 independent simulations are completed and the median of the outcomes is shown in Fig. 11. The results show that the resultant demodulators can provide an acceptable BER performance for initial start up such that the receiver can be switched into the decision mode and adapted further for better BER performance. When the timing error is introduced in acquisition, the MOE algorithm with constraints provides a better BER performance because the constraints can avoid the problem of cancelling the desired signal

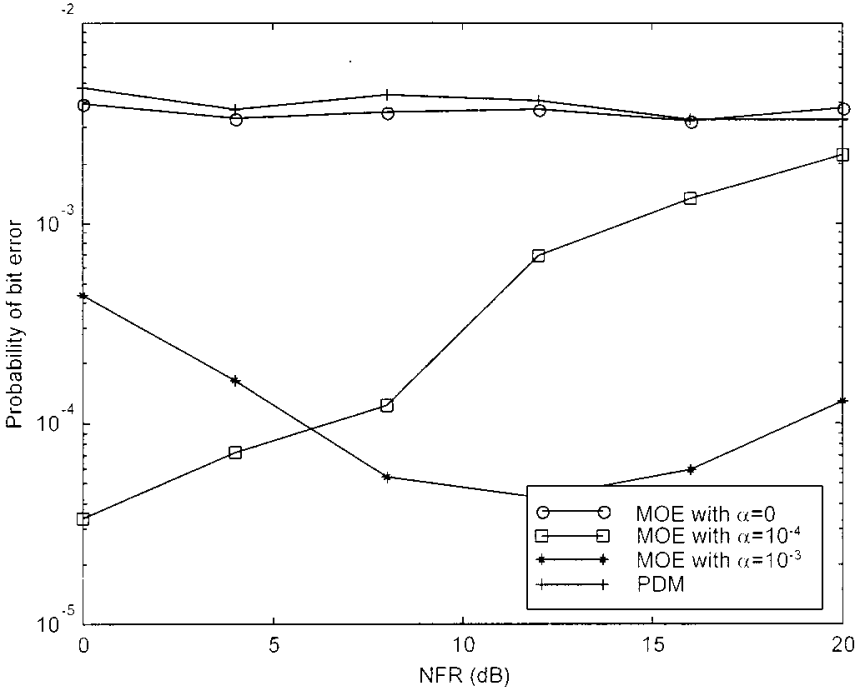

Fig. 11. BER of the resultant demodulators versus NFR

and noise enhancement. This fact is confirmed by that the resultant demodulators derived by the MOE algorithm without constraints and the PDM algorithm have the similar performance of bit error probability.

\section{CONCLUSION}

In this paper, we have presented an efficient blind algorithm for estimating the code timing of a desired user in an asynchronous DS/CDMA system. After the code timing is estimated explicitly, the MMSE linear detector can be obtained. Numerical results show that the proposed algorithm is near-far resistant. Furthermore, the proposed algorithm is more robust to the multipath interference compared to other timing estimators.

\section{APPENDIX A}

Proof of Lemma: Let the unit-norm vector $\mathbf{u}_{l}$ lie in the signal subspace spanned by the eigenvectors of $\mathbf{R}$ with respect to the $L$ largest eigenvalues, which can be expressed as

$$
\mathbf{u}_{l}=\sum_{n=1}^{L} \alpha_{n} \mathbf{q}_{n}
$$

where $\sum_{n=1}^{L} \alpha_{n}^{2}=1$. After some manipulations, we have

$$
\begin{aligned}
\mathbf{u}_{l}^{T} \mathbf{R}^{-1} \mathbf{u}_{l} & =\sum_{n=1}^{L} \frac{\alpha_{n}^{2}}{\gamma_{n}+\sigma^{2}} \\
& \leq \frac{1}{\gamma_{L}+\sigma^{2}}
\end{aligned}
$$

because of $\gamma_{1} \geq \gamma_{2} \geq \cdots \geq \gamma_{L}$. Assuming that $\mathbf{c}$ is an any vector of length $2 N$ with unit norm. It can be expressed in terms of linearly combining $\mathbf{q}_{n}$ as follows:

$$
\mathbf{c}=\sum_{n=1}^{2 N} \beta_{n} \mathbf{q}_{n}
$$


where $\sum_{n=1}^{2 N} \beta_{n}^{2}=1$. Following the same way, we can obtain

$$
\begin{aligned}
\mathbf{c}^{T} \mathbf{R}^{-1} \mathbf{c} & =\sum_{n=1}^{L} \frac{\beta_{n}^{2}}{\gamma_{n}+\sigma^{2}}+\sum_{n=L+1}^{2 N} \frac{\beta_{n}^{2}}{\sigma^{2}} \\
& \geq \sum_{n=1}^{L} \frac{\beta_{n}^{2}}{\gamma_{1}+\sigma^{2}}+\sum_{n=L+1}^{2 N} \frac{\beta_{n}^{2}}{\sigma^{2}} \\
& =\frac{z}{\gamma_{1}+\sigma^{2}}+\frac{1-z}{\sigma^{2}}
\end{aligned}
$$

where $\sum_{n=1}^{L} \beta_{n}^{2}=z$ and $\sum_{n=L+1}^{2 N} \beta_{n}^{2}=1-z$. Subtracting $1 /\left(\gamma_{L}+\sigma^{2}\right)$ from $z /\left(\gamma_{1}+\sigma^{2}\right)+(1-z) / \sigma^{2}$, we have the numerator given by

$$
\text { Num }=\gamma_{1} \gamma_{L}(1-z)+\sigma^{2}\left(\gamma_{L}-z \gamma_{1}\right) \text {. }
$$

When SNR is high enough (i.e., $\sigma^{2}$ is sufficiently small), the second term can be negligible and the numerator is always positive. The proof is thus completed.

\section{APPENDIX B}

The details of interpolating the two best hypotheses and determining the delay of interest are supplied here. Suppose $H_{p}$ and $H_{p+1}$ are the two best hypotheses. The corresponding nominal desired signal vectors can be expressed as

$$
\begin{aligned}
\text { when } p \text { is even }\left(n=\frac{p}{2}\right): & \mathbf{c}_{1}=\frac{T_{R}^{n} \mathbf{a}_{1}}{\left\|T_{R}^{n} \mathbf{a}_{1}\right\|} \\
& \mathbf{c}_{2}=\frac{T_{R}^{n-1} \mathbf{a}_{1}+T_{R}^{n} \mathbf{a}_{1}}{\left\|T_{R}^{n-1} \mathbf{a}_{1}+T_{R}^{n} \mathbf{a}_{1}\right\|} \\
\text { when } p \text { is odd }\left(n=\frac{p-1}{2}\right): & \mathbf{c}_{1}=\frac{T_{R}^{n} \mathbf{a}_{1}}{\left\|T_{R}^{n} \mathbf{a}_{1}\right\|} \\
\mathbf{c}_{2} & =\frac{T_{R}^{n+1} \mathbf{a}_{1}+T_{R}^{n} \mathbf{a}_{1}}{\left\|T_{R}^{n+1} \mathbf{a}_{1}+T_{R}^{n} \mathbf{a}_{1}\right\|} .
\end{aligned}
$$

The interpolated nominal can be given by

$$
\mathbf{c}_{\text {out }}=(1-\mu) \mathbf{c}_{2}+\mu \mathbf{c}_{1}=\mathbf{C e}
$$

where $\mathbf{C}=\left[\mathbf{c}_{2} \mathbf{c}_{1}\right]$ and $\mathbf{e}=[1-\mu \mu]^{T}$, in which $\mu \in[0,1]$ is determined to minimize the projection degree measurement given by $\xi(\mu)=\left(\mathbf{c}_{\text {out }}^{T} \mathbf{R}^{-1} \mathbf{c}_{\text {out }}\right) /\left(\left\|\mathbf{c}_{\text {out }}\right\|^{2}\right)$.

The numerator of $\xi(\mu)$ can be expressed as $N(\mu)=\mathbf{e}^{T} \mathbf{G e}$, where $\mathbf{G}=\mathbf{C}^{T} \mathbf{R}^{-1} \mathbf{C}$. Similarly, the denominator of $\xi(\mu)$ can be expressed as $D(\mu)=\mathbf{e}^{T} \mathbf{H e}$, where $\mathbf{H}=\mathbf{C}^{T} \mathbf{C}$. Since the numerator and denominator are quadratic in $\mu$, any extreme point $\mu$ must satisfy the following equation $S(\mu)=N^{\prime}(\mu) D(\mu)-N(\mu) D^{\prime}(\mu)=0$. Furthermore, $N(\mu)$ and $D(\mu)$ can be written as

$$
\begin{aligned}
N(\mu)= & {\left[G_{11}+G_{22}-G_{12}-G_{21}\right] \mu^{2} } \\
& +\left[G_{12}+G_{21}-2 G_{11}\right] \mu+G_{11} \\
= & n_{2} \mu^{2}+n_{1} \mu+n_{0} \\
D(\mu)= & {\left[H_{11}+H_{22}-H_{12}-H_{21}\right] \mu^{2} } \\
& +\left[H_{12}+H_{21}-2 H_{11}\right] \mu+H_{11} \\
= & d_{2} \mu^{2}+d_{1} \mu+d_{0} .
\end{aligned}
$$

Therefore, we have

$$
\begin{aligned}
S(\mu) & =\left(n_{2} d_{1}-n_{1} d_{2}\right) \mu^{2}+2\left(n_{2} d_{0}-n_{0} d_{2}\right) \mu+n_{1} d_{0}-n_{0} d_{1} \\
& =s_{2} \mu^{2}+s_{1} \mu+s_{0} .
\end{aligned}
$$

The roots to $S(\mu)$ are

$$
\mu_{1}, \mu_{2}=\frac{1}{2 s_{2}}\left[-s_{1} \pm \sqrt{s_{1}^{2}-4 s_{2} s_{0}}\right] .
$$

Therefore, we choose $\mu_{\min }$ to be the value which minimizes $\xi(\mu)$ over $\mu=1,0$ and either of $\mu_{1}, \mu_{2}$ that fall in $[0,1]$. After $\mu_{\min }$ is chosen, the estimated timing can be expressed as

$$
\begin{array}{ll}
\hat{\tau}_{1} / T_{c}=n-\frac{a}{a+b}, & p \text { is even } \\
\hat{\tau}_{1} / T_{c}=n+\frac{a}{a+b}, & p \text { is odd }
\end{array}
$$

where $a=\left(\mu_{\min } /\left\|\mathbf{c}_{1}\right\|\right)+\left(\left(1-\mu_{\min }\right) /\left\|\mathbf{c}_{2}\right\|\right)$ and $b=(1-$ $\left.\mu_{\min }\right) /\left\|\mathbf{c}_{2}\right\|$.

\section{REFERENCES}

[1] R. E. Ziemer and R. L. Peterson, Digital Communications and Spread Spectrum Systems. New York: Macmillan, 1985.

[2] U. Madhow and M. L. Honig, "MMSE interference suppression for direct-sequence spread spectrum CDMA,” IEEE Trans. Commun., vol. 42, pp. 3178-3188, Dec. 1994.

[3] S. L. Miller, "An adaptive direct-sequence code-division multiple-access receiver for multiuser interference rejection," IEEE Trans. Commun., vol. 43, pp. 1746-1755, Feb./Mar./Apr. 1995.

[4] A. N. Barbosa and S. L. Miller, "Adaptive detection of DS/CDMA signals in fading channels," IEEE Trans. Commun., vol. 46, pp. 115-124, Jan. 1998.

[5] M. Honig, U. Madhow, and S. Verdu, "Blind adaptive interference suppression for near-far resistant CDMA," in Proc. 1994 Globecom, pp 379-384.

[6] - "Blind adaptive multiuser detection," IEEE Trans. Inform. Theory, vol. 41, pp. 944-960, 1995.

[7] J. B. Schodorf and D. B. Williams, "A constrained optimization approach to multiuser detector," IEEE Trans. Signal Processing, vol. 45 , pp. 258-262, 1997.

[8] N. Zecevic and J. H. Reed, "Blind adaptation algorithms for direct-sequence spread spectrum CDMA single-user detection," in IEEE Veh. Technol. Conf., 1997, pp. 2133-2137.

[9] J. Li and S. Tantaratana, "Optimal and suboptimal coherent acquisition schemes for PN sequences with data modulation," IEEE Trans. Commun., vol. 43, pp. 554-564, Feb./Mar./Apr. 1995.

[10] R. F. Smith and S. L. Miller, "Code timing estimation in a near-far environment for direct-sequence code-division multiple-access," in Proc Milcom'94, pp. 47-51.

[11] E. G. Strom, S. Parkvall, S. L. Miller, and B. E. Ottersten, "Propagation delay estimation in asynchronous direct-sequence code-division multiple access systems," IEEE Trans. Commun., vol. 44, pp. 84-93, Jan. 1996.

[12] S. E. Bensley and B. Aazhang, "Subspace-based channel estimation for code division multiple access communication systems," IEEE Trans. Commun., vol. 44, pp. 1009-1020, Aug. 1996.

[13] U. Madhow, "Adaptive interference suppression for joint acquisition and demodulation of direct-sequence CDMA signals," in Proc. Milcom'95, pp. 1200-1204.

[14] —, "Blind adaptive interference suppression for the near-far resistant acquisition and demodulation of direct-sequence CDMA signals," IEEE Trans. Signal Processing, vol. 45, pp. 124-136, 1997.

[15] D. Zheng, J. Li, S. L. Miller, and E. G. Strom, "An efficient code-timing estimator for DS-CDMA signals," IEEE Trans. Signal Processing, vol. 45, pp. 82-89, 1997.

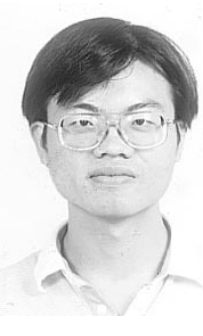

Ho-Chi Hwang (S'93-M'99) was born in Taiwan, R.O.C., in 1969. He received the B.S., M.S., and $\mathrm{Ph} . \mathrm{D}$. degrees in electronics engineering from National Chiao Tung University, Hsin-Chu, Taiwan, in 1991, 1993, and 1999, respectively.

$\mathrm{He}$ is currently a Researcher at the Computer \& Communications Research Laboratories, Industrial Technology Research Institute, Taiwan. His research interests include digital communications, interference suppression in CDMA communications, and adaptive signal processing. 


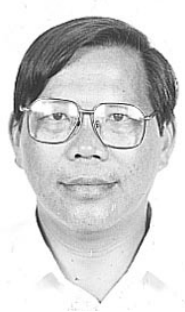

Che-Ho Wei (S'73-M'76-SM'87-F'96) was born in Taiwan, R.O.C., in 1946. He received the B.S. and M.S. degrees in electronics engineering from National Chiao Tung University, Hsin-Chu, Taiwan, in 1968 and 1970, respectively, and the Ph.D. degree in electrical engineering from the University of Washington, Seattle, in 1976.

From 1976 to 1979, he was an Associate Professor at National Chiao Tung University, where he is now a Professor with the Department of Electronics Engineering and Dean of the College of Electrical Engineering and Computer Science. From 1979 to 1982, he was the Engineering Manager of Wang Industrial Company, Taipei, Taiwan. He was the Chairman of the Department of Electronics Engineering, National Chiao Tung University, from 1982 to 1986 and Director of the Institute of Electronics from 1984 to 1989.

He was on leave from the Ministry of Education and served as the Director of the Advisory Office from September 1990 to July 1992. His research interests include digital communications and signal processing and related VLSI circuits design. 